Monatsschr Kinderheilkd 2011 · 159:426-426

DOI 10.1007/s00112-010-2331-0

(c) Springer-Verlag 2011

\author{
F. Zepp ${ }^{1} \cdot$ V. Wahn ${ }^{2}$ \\ ${ }^{1}$ Zentrum für Kinder- und Jugendmedizin, Universitätsmedizin Mainz \\ ${ }^{2}$ Klinik für Pädiatrie mit Schwerpunkt Pneumologie und Immunologie, \\ Charité Campus Virchow, Berlin
}

\title{
Das infektanfällige Kind
}

In den vergangenen 10 Jahren widmete die Monatsschrift Kinderheilkunde dem Thema Infektanfälligkeit und Immundefekt bereits 2-mal ein Schwerpunktheft. Die klinische Bedeutung des Leitsymptoms, aber auch der enorme Wissenszuwachs der vergangenen Jahre, sowohl hinsichtlich der molekularen Grundlagen als auch neuer therapeutischer Konzepte von Immunfunktionsstörungen, veranlasste die Herausgeber, die aktuellen Erkenntnisse jetzt erneut in den Mittelpunkt eines Themenheftes zu stellen.

Das wiederholte Auftreten von Infektionen, meist der Atemwege, ist im Kindesalter nicht grundsätzlich ungewöhnlich. Infektionskrankheiten sind, gerade in der kühlen Jahreszeit, der häufigste Anlass für die Vorstellung von Kindern in kinderärztlichen Praxen und Kliniken. Wann aber handelt es sich bei diesen Symptomen um eine pathologische Häufung von Infekten, die möglicherweise auf eine prädisponierende Störung der Abwehrfunktionen hinweist? Der Beitrag von Baumann und Niehues hat die Präzisierung des Begriffs Infektanfälligkeit zum Ziel, insbesondere im Hinblick auf die Frage, welche anamnestischen und klinischen Befunde Hinweise auf das Vorliegen einer immunologischen Störung oder eines Immundefekts geben. Einfache Untersuchungen können dabei in der Praxis hilfreich sein, für besondere Problemsituationen oder Spezialdiagnostik wird die Vernetzung mit einem pädiatrischen Immundefektzentrum empfohlen. Unterstützung können Kinderärzte/-ärztinnen auch über Aktivitäten wie das FIND-ID-Projekt finden, eine interdisziplinäre Initiative, die die Suche nach einem fachkompetenten Gesprächspartner unterstützen will. Ein wichtiges Ziel ist es, für Frühsymptome einer immunologischen Störung zu sensi- bilisieren. Kein Kind, bei dem ein Immundefekt vorliegt, sollte heute zu spät erkannt werden oder gar unentdeckt bleiben.

\section{$>$ Kein Kind mit Immundefekt sollte heutzutage zu spät identifiziert werden}

Bei Verdacht auf einen Immundefekt ist ein strukturiertes diagnostisches Vorgehen, das sich an der klinischen Symptomatik orientiert, unerlässlich. Von Bernuth et al. illustrieren anhand ausgewählter Fallbeispiele ein diagnostisches Stufenkonzept, das von der Basisdiagnostik in der Praxis zur spezifischen immunologischen und molekulargenetischen Analyse in Spezialzentren führt. Natürlich ist es bei vermuteten genetischen Erkrankungen auch ein Ziel, die verantwortliche Mutation zu identifizieren. Die präzise genetische Diagnose ermöglicht die Beratung der Familie, ggf. die Planung einer Pränataldiagnostik, und in Zukunft vielleicht auch die somatische Gentherapie.

Die frühe Diagnose eines Immundefekts ist unerlässlich, um irreversible Organschäden oder frühzeitigen Tod zu vermeiden. Erfreulicherweise stehen heute Behandlungsoptionen zur Verfügung, die bei den betroffenen Kindern Morbidität und Letalität drastisch verringern und ihnen ein weitgehend normales Leben ermöglichen. Das Spektrum der therapeutischen Möglichkeiten wird in dem Beitrag von Wahn et al. zusammenfassend dargestellt.

Die deutschen Kinder- und Jugendärzte tragen durch die konsequente Umsetzung der Impfungen im Kindes- und Jugendalter entscheidend dazu bei, schwerwiegende Infektionen und deren Komplikationen zu verhindern. Dies kann als großer Erfolg der präventiven Pädiatrie gewertet werden. Besondere Regeln gelten jedoch für immundefiziente Kinder, bei denen auch bestimmte Impfrisiken berücksichtigt werden müssen. Umfassend beschreiben Mannhardt und Wahn den Nutzen und die Risiken der Impfungen in dieser besonderen Gruppe von $\mathrm{Pa}$ tienten und geben wichtige Hinweise für den klinischen Alltag.

Für die Herausgeber dieses Leitthemas war es eine besondere Freude, hervorragende Autoren/-innen mit großer fachlicher Kompetenz für die Mitarbeit zu gewinnen. Alle wurden ihrer Aufgabe mit großer Sorgfalt gerecht und allen sei für ihr Engagement an dieser Stelle ganz herzlich gedankt.

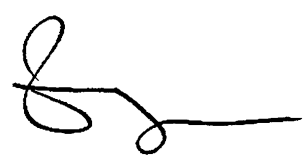

Prof. Dr. Fred Zepp

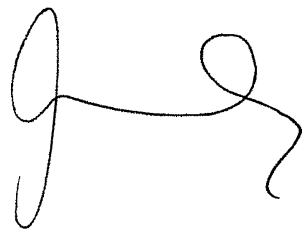

Prof. Dr. Volker Wahn

\section{Korrespondenzadresse \\ Prof. Dr. V. Wahn \\ Charité Campus Virchow \\ Klinik m.S. Pädiatrische Pneumologie \\ und Immunologie \\ Augustenburger Platz 1, 13353 Berlin \\ volker.wahn@charite.de \\ Prof. Dr. F.Zepp \\ Johannes-Gutenberg-Universität \\ Kinderklinik und Kinderpoliklinik \\ Czernyweg. 55131 Mainz \\ Zepp@Kinder.Klinik.uni-mainz.de}

